\title{
REFERENCES
}

1. E. A. Robinson, Sums of stationary random variables, Proc. Amer. Math. Soc. vol. 11 (1960) pp. 77-79.

2. J. L. Doob, Stochastic processes, New York, Wiley, 1953.

John Jay Hopkins Laboratory for Pure and Applied Science, General Atomic Division of General Dynamics Corporation, San Diego, California

\section{APPROXIMATION OF INTEGRAL CURRENTS BY CYCLES ${ }^{1}$}

\section{HERBERT FEDERER}

The purpose of this note is to prove the following theorem concerning isoperimetric ratios and cyclic approximation:

If $A$ and $B$ are compact Lipschitz neighborhood retracts in $R^{n}$, with $A \supset B$, and $k$ is a positive integer then, there exists a positive number $\rho$ with the following two properties:

(1) For each $X \in \partial I_{k+1}(A)+I_{k}(B)$ there exists a $Y \in I_{k+1}(A)$ such that $\operatorname{spt}(X-\partial Y) \subset B$ and

$$
\boldsymbol{M}(Y)^{k /(k+1)} \leqq \rho\|X\|(A-B) .
$$

(2) For each $T \in I_{k+1}(A)$ there exists an $S \in I_{k+1}(A)$ such that spt $\partial S$ $\subset B$ and

$$
M(T-S)^{k /(k+1)} \leqq \rho\|\partial T\|(A-B) .
$$

The notation is the same as in Normal and integral currents by H. Federer and W. H. Fleming, Ann. of Math. vol. 72 (1960) pp. 458-520, and all references (like 6.1 below) are to that paper.

Clearly (2) follows from (1), with $X=\partial T, S=T-Y$.

To prove (1) let $U, V, f, g, a, b, \xi, \eta, c_{1}, c_{2}, c_{3}$ be as in 6.1 with $\Gamma=A$, let

$$
\begin{aligned}
\epsilon & =\inf \{a /[3 n(\eta+1)], b /(3 n)\} \\
\Phi & =R^{n} \cap\{x: \text { distance }(x, A) \leqq 3 n \epsilon\}, \\
\Psi & =R^{n} \cap\{x: \text { distance }(x, B) \leqq 3 n \epsilon\},
\end{aligned}
$$

let $K$ be the subcomplex of $\mu_{\epsilon}\left(C^{\prime}\right)$ (see $2.1,5.1$ ) consisting of the cubes contained in $\Phi$, and let $\Omega$ be the group of those $k$ dimensional chains of $K$ which belong to

Presented to the Society, April 16, 1960; received by the editors December 2, 1960.

1 This work was supported in part by a Sloan Fellowship. 


$$
\partial I_{k+1}(\Phi)+I_{k}(\Psi) .
$$

Then $\Omega$ has a finite number of free generators $\omega_{1}, \cdots, \omega_{m}$ and one may choose

$$
\chi_{i} \in I_{k+1}(\Phi) \text { with } \operatorname{spt}\left(\omega_{i}-\partial \chi_{i}\right) \subset \Psi
$$

for $i=1, \cdots, m$. Furthermore $\omega_{1}, \cdots, \omega_{m}$ form a base for a vectorsubspace $W$ of $\boldsymbol{E}_{k}(\Phi)$ normed by $\boldsymbol{M}$, and the linear transformation

$$
L: W \rightarrow N_{k+1}(\Phi)
$$

such that $L\left(\omega_{i}\right)=\chi_{i}$ for $i=1, \cdots, m$ has the finite norm

$$
\lambda=\sup \{\boldsymbol{N}[L(w)]: w \in W, \boldsymbol{M}(w) \leqq 1\} .
$$

Finally let

$$
\begin{aligned}
& \tau=c_{1}^{-1}\left[\inf \left\{b,(\eta+2)^{-1} a\right\}\right]^{k}, \\
& \sigma=\sup \left\{2 n^{k}\left(\begin{array}{l}
n \\
k
\end{array}\right), 6 n^{k}\left(\begin{array}{c}
n \\
k-1
\end{array}\right), 4 n^{k+1}\left(\begin{array}{l}
n \\
k
\end{array}\right) \epsilon\right\}, \\
& \zeta=\sigma+2 \sigma \lambda+(\eta+1) 3 n \epsilon \eta^{k}(1+4 \sigma+2 \sigma \lambda), \\
& \rho=\sup \left\{c_{2} \xi^{k}(\eta+2)^{k}, \xi^{k} \xi^{k /(k+1)} \tau^{-1 /(k+1)}\right\} .
\end{aligned}
$$

Now suppose $X \in \partial I_{k+1}(A)+I_{k}(B)$.

In case $\|X\|(A-B) \leqq \tau$, the conclusion of (1) follows from 6.1. Henceforth assume that $\|X\|(A-B)>\tau$, let

$$
X_{s}=X \cap\{x \text { : distance }(x, B)>s\} \text { for } s>0,
$$

use 3.10 with the fact that spt $\partial X \subset B$ to obtain

$$
\int_{0}^{\bullet} M\left(\partial X_{s}\right) d s \leqq\|X\|(A-B),
$$

and choose a number $s$ for which

$$
0<s<\epsilon, \quad M\left(\partial X_{s}\right) \epsilon \leqq\|X\|(A-B) .
$$

Noting that

$$
\text { spt } \partial X_{s} \subset R^{n} \cap\{x: \text { distance }(x, B)=s\}
$$

and applying 5.5 with $T=X_{s}$ one finds that

$$
\begin{aligned}
X_{s} & =P+Q+\partial S, \quad Q \in I_{k}(\Psi), \quad S \in I_{k+1}(\Phi), \\
P & =X+\left(X_{s}-X\right)-Q-\partial S \in \Omega, \\
M(P) & \leqq 2 \sigma\|X\|(A-B), \quad \boldsymbol{M}(Q) \leqq \sigma\|X\|(A-B), \\
M(S) & \leqq \sigma\|X\|(A-B), \quad \boldsymbol{M}(\partial S) \leqq 4 \sigma\|X\|(A-B) .
\end{aligned}
$$


Accordingly

$$
\begin{aligned}
L(P) & \in I_{k+1}(\Phi), \quad \operatorname{spt}[P-\partial L(P)] \subset \Psi, \\
N[L(P)] & \leqq \lambda M(P) \leqq 2 \sigma \lambda\|X\|(A-B), \\
\Xi & =X-\partial S-\partial L(P) \\
& =\left(X-X_{\diamond}\right)+Q+[P-\partial L(P)] \in I_{k}(\Psi), \\
\|\Xi\|(\Psi-B) & \leqq\|X\|(A-B)+M(\partial S)+N[L(P)] \\
& \leqq(1+4 \sigma+2 \sigma \lambda)\|X\|(A-B),
\end{aligned}
$$

with $\partial \Xi=\partial X \in I_{k-1}(B)$. Letting $h$ be the linear homotopy from $g$ to the identity map, and noting that

$$
g(x)=x \text { for } x \in B, \quad|g(x)-x| \leqq(\eta+1) 3 n \in \text { for } x \in \Psi,
$$

one obtains

$$
\begin{aligned}
\Xi-g_{\boldsymbol{f}}(\Xi) & =\partial h_{\boldsymbol{t}}(I \times \Xi), \\
M\left[h_{\boldsymbol{t}}(I \times \Xi)\right] & \leqq(\eta+1) 3 n \epsilon \eta^{k}\|\Xi\|(\Psi-B), \\
X-g_{\boldsymbol{f}}(\Xi) & =\partial\left[S+L(P)+h_{\boldsymbol{t}}(I \times \Xi)\right], \\
M[S+L(P) & \left.+h_{\boldsymbol{f}}(I \times \Xi)\right] \leqq \zeta\|X\|(A-B) .
\end{aligned}
$$

Inasmuch as

$\Phi \cup h(I \times \Psi) \subset R^{n} \cap\{x:$ distance $(x, A) \leqq a\}$, one may define

$$
Y=f_{+}\left[S+L(P)+h_{+}(I \times \Xi)\right] \in I_{k+1}(A)
$$

and conclude that

$$
\begin{aligned}
X-g_{f}(\Xi) & =f_{f}\left[X-g_{f}(\Xi)\right]=\partial Y, \\
M(Y) & \leqq \xi^{k+1} \xi\|X\|(A-B) \\
& \leqq \xi^{k+1} \zeta \tau^{-1 / k}[\|X\|(A-B)]^{(k+1) / k}, \\
M(Y)^{k /(k+1)} & \leqq \rho\|X\|(A-B) .
\end{aligned}
$$

BROWN UNIVERSITY 